\title{
Fuelling efficiency of massive gas injection in TEXTOR. Mass scaling and importance of gas flow dynamics.
}

\author{
S A Bozhenkov ${ }^{1}$, M Lehnen ${ }^{2}$, K H Finken ${ }^{2}$, G Bertschinger ${ }^{2}$, H \\ R Koslowski ${ }^{2}$, D Reiter ${ }^{2}, \mathbf{R}$ C Wolf ${ }^{1}$ and TEXTOR team \\ ${ }^{1}$ Max-Planck-Institut für Plasmaphysik, D-17491 Greifswald, Germany \\ ${ }^{2}$ Institute for Energy Research - Plasma Physics, Forschungzentrum Jülich GmbH, \\ EURATOM-Association, Trilateral Euregio Cluster, D-52425 Juelich, Germany \\ E-mail: sergey.bozhenkov@ipp.mpg.de
}

\begin{abstract}
.
Fuelling efficiency is an important parameter in designing a massive gas injection system for suppression of runaway electrons in ITER. In this work a $Z$-dependence of the fuelling efficiency is measured for TEXTOR. The dependence covers the following gases: $\mathrm{He}, \mathrm{Ne}, \mathrm{Ar}, \mathrm{Kr}, \mathrm{Xe}$ and a $10 \%$ Ar- $\mathrm{D}_{2}$ mixture. It is shown that the fuelling efficiency significantly decreases with the gas mass, from above 0.5 for He to below 0.03 for Xe.

To explain the variation of the efficiency with the gas mass and pressure a simple model of the gas flow from the valve to the plasma edge is developed. The flow model is validated by using available laboratory flow measurements of a TEXTOR-similar injection system. The unsteady gas flow and a premature plasma disrupting are shown to explain the mass dependence of the efficiency.
\end{abstract}




\section{Introduction}

Tokamak disruptions are fast events resulting in a total loss of the discharge energy: both the thermal and magnetic energy is dissipated. Loads on a future fusion machine due to such an abrupt energy release are of particular concern. Therefore, the ITER project [1] requires a reliable disruption mitigation technique [2]. The criteria of a successful mitigation are: (i) reduction of the heat loads during the thermal quench; (ii) reduction of the halo currents; (iii) and suppression of runaway electrons (RE). The first two criteria can be met either with massive gas injection (MGI) [3-5] or with killer pellets [6-8], while the RE suppression turns out to be more challenging.

To achieve collisional runaway suppression, the total electron density must be rapidly increased by several orders of magnitude. Being able to inject at least hundred times more particles than the killer pellet method, massive gas injection seems to be the most suitable technique for this increase. For the ITER design, the fuelling efficiency of MGI and its scaling are of importance. It is to be mentioned that a novel shattered pellet technique significantly improves the number of atoms delivered by a pellet [9-11], the scalability of this technique is also under investigation.

Experiments with MGI in TEXTOR earlier indicated that the fuelling efficiency before the current quench is rather low for medium- $Z$ gases [12]. Specifically, the efficiency was found to be 0.4 for helium, 0.15 for a $10 \%$ argon-deuterium mixture, and 0.03 for argon. In this work the $Z$-dependence is extended to include neon, krypton and xenon and the old data are slightly refined. To explain the efficiency variation with the gas mass and pressure a simple model of the gas flow from the valve to the plasma edge is proposed. The flow model is validated by using available laboratory flow measurements of a TEXTOR-similar injection system $[13,14]$. The unsteady gas flow and a premature plasma disrupting are shown to explain the mass dependence: the gas pulse is smeared out and a small fraction of atoms at its front causes the disruption before the bulk can reach the plasma. Because both the smearing and the cooling capability increase with the gas, it is increasingly difficult to deposit a large number of high- $Z$ atoms.

An overview of disruptions, their consequences and possible mitigation schemes is given in $[15,16]$ and the references therein. Physics of runaway electrons, possible methods of their suppression and runaway current termination are discussed in $[2,12,17-19,41-43]$. Here we consider only collisional runaway suppression and limit ourselves to a brief explanation of why the electron density must be raised extremely for the suppression to be guarantied.

Runaway electrons appear, if an electric field $E$ is applied to plasma, because the drag force $\mathrm{F}$ exerted by Coulomb collisions decreases with the relative velocity (energy). In the relativistic limit, however, the drag has a minimum. As a consequence, runaways 
can not appear, if the field $E$ is too small $[20,21]$ :

$$
E \leq E_{C}=\frac{\min (\mathrm{F})}{e}=\frac{4 \pi e^{3} \ln \Lambda}{m c^{2}} n_{e}^{*} \approx 5.2 \cdot 10^{-22} \cdot n_{e}^{*} \mathrm{~V} / \mathrm{m},
$$

where the total electron density $n_{e}^{*}$ is in $\mathrm{m}^{-3}$ and includes bound electrons. Since the electric field depends on the density of introduced impurities, to be read the total electron density, the condition has to be calculated self consistently, which has not been done yet. For the sake of simplicity, one might assume for $E$ a typical value observed in the present-day machines, e.g. the value of $50 \mathrm{~V} / \mathrm{m}$ results in the density of above $10^{23} \mathrm{~m}^{-3}$. This density exceeds by three orders of magnitude the electrons density in an ITER steady state plasma. A typical time scale for raising the density is of the order of a few milliseconds.

At present, experiments with MGI are conducted at several tokamaks: DIII-D, ASDEX Upgrade, TEXTOR and JET. But there is no uniform definition of the fuelling efficiency.

At DIII-D the assimilation or mixing efficiency $Y(t)$ is defined as the ratio of the plasma electron density measured along a central chord and multiplied by the plasma volume to the average charge state and to the number of particles delivered until this time [22]. To have a figure-of-merit $\bar{Y}$, the assimilation is averaged between the value measured at the beginning and at the middle of the current quench. This average assimilation is found to be independent of the injected species for $\mathrm{H}_{2}, \mathrm{D}_{2}$, Ne and $\mathrm{Ar}$ and to vary between 5 and $20 \%$ for different plasma parameters. Surprisingly, a two times higher value is found for He.

At ASDEX Upgrade the fuelling efficiency is defined as the ratio of the electron density increase multiplied by the plasma volume to the total number of injected atoms or molecules [5]. The electron density is averaged over a central and an edge interferometer chords and from the thermal quench until the plasma current drops to the $20 \%$ level. The fuelling efficiency increased by a factor of $2-3$ after the valve-plasma distance had been reduced from $1.5 \mathrm{~m}$ to $10 \mathrm{~cm}$. There is a strong dependence of the efficiency on the gas mass: about $40 \%$ for He, about $20 \%$ for Ne and below 10\% for Ar. For all gases the efficiency decreases as the number of particles is increased. A dependence on the plasma thermal energy is not clear, but the efficiency seems to descend as the plasma energy grows.

At JET the fuelling efficiency is introduced as the ratio of the number of atoms in the plasma at the current quench to the total number of atoms stored initially in the injecting system [23]. The number of atoms in the plasma is extracted from a model of the current decay. The JET efficiency decreases from about $25 \%$ for a $10 \%$ mixture of Ne with $\mathrm{D}_{2}$ to $5 \%$ for $\mathrm{Ne}$ and $2.5 \%$ for Ar. 
At TEXTOR previously [12] the mixing efficiency was defined as the ratio of the number of atoms found in the plasma at the current quench to the total number of released atoms. The number of atoms in the plasma was determined by using a simple model of the current quench. The term "mixing efficiency" is not the most suitable here, for not all released atoms reach the plasma before the disruption. A more suitable name seems indeed to be "fuelling efficiency", defined in the same way as at JET. This name is accepted throughout this work, while the mixing efficiency is understood as the ratio of the fuelling efficiency to the relative number of particles delivered to the plasma before disruption, i.e. the latter measures mixing of atoms into the plasma core during thermal quench.

The accepted definition of fuelling efficiency shows capability of an injection scheme to deposit a desired number of atoms before the current quench onset. The definition uses number of atoms in plasma instead of free electron density, because the runaway suppression condition (equation 1) contains both bound and free electrons. The number of atoms in plasma is taken at the start of the current quench, that is at the moment when the flux surfaces are thought to be healed and generation of runaway electrons is possible. The RE suppression at this moment is the most favorable one, for it does not leave any possibilities for a strong beam generation. Besides, experiments in DIII-D [22] indicate that the particle assimilation during current quench is not effective. Fuelling efficiency is also easy to use as a design parameter, for example for ITER. It is to be noted that an alternative mitigation scenario is possible, where the critical density for RE suppression is achieved later in the current quench in such a way that the resulting runaway avalanche can still be tolerated by the machine. An analysis of this alternative scenario is beyond the scope of the paper.

The rest of the article is organized in the following way: in section 2 a model of the gas flow is developed; the model is validated in section 3 ; in section 4 TEXTOR measurements of the fuelling efficiency are described and the results are compared to the flow model; and finally in section 5 the results are summarized and possible implications are outlined.

\section{Gas flow model}

In MGI experiments a gas reservoir pre-filled to a high initial pressure is quickly opened to release the particles through a vacuum delivery tube. Opening times of the used injectors are much shorter than the injection duration, e.g. for the TEXTOR system the opening is of the order of a few hundreds of microseconds [24]. The problem is to find flow parameters at the plasma edge. A simplified problem of relevance is one dimensional expansion of an uniform gas into vacuum in an infinite tube. In this section the solution of the simplified problem is presented. The solution describes effects re- 


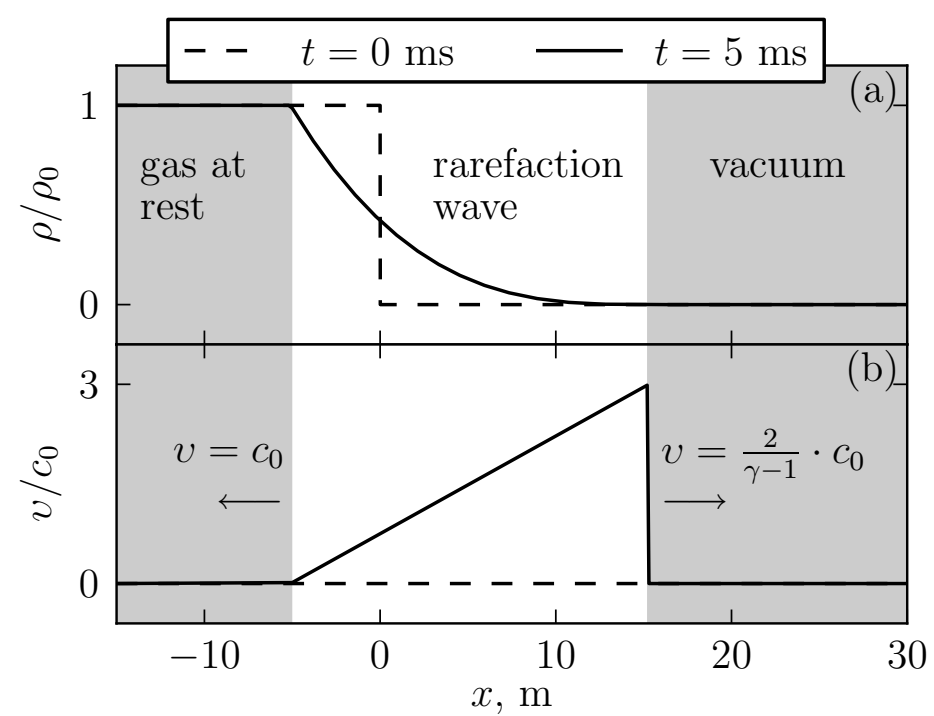

Figure 1. One dimensional unsteady gas expansion $\left(\mathrm{He}, T_{0}=300 \mathrm{~K}\right)$. Distributions of the density (a) and gas velocity (b) along the $x$-axis are plotted for two times. On the left the undisturbed gas is highlighted. On the right the vacuum region is indicated. The middle part is occupied by the rarefaction wave. The wave head runs to the left with the sound speed $c_{0}$. The wave tail is the gas-vacuum interface running to the right with the velocity of $\frac{2}{\gamma-1} \cdot c_{0}$.

lated to the flow non-stationarity but neglects many real factors, like real piston motion, turbulence, condensation, viscosity. Application of the idealized model is justified by a laboratory calibration discussed later.

Initially, an infinite tube is separated by a diaphragm at position $x=0$. On the left the tube is uniformly filled with gas, at the right it is evacuated to vacuum. This situation is shown with a dashed line in figure 1 . At time $t=0$ the diaphragm is instantaneously removed, the resulting flow is described by Euler equations:

$$
\begin{aligned}
& \frac{\partial \rho}{\partial t}+\frac{\partial(\rho u)}{\partial x}=0 \\
& \frac{\partial v}{\partial t}+v \frac{\partial v}{\partial x}=-\frac{1}{\rho} \frac{\partial p}{\partial x} \\
& p \cdot \rho^{-\gamma}=\text { const }
\end{aligned}
$$

where $\gamma$ is the ratio of the specific heats $c_{p} / c_{v}$. The system can be solved analytically at least by two methods: by exploiting characteristics and Riemann invariants [25-28], or by using self-similarity of the problem $[25,27]$. The structure of the solution known as centered rarefaction wave is illustrated with distributions of the density and velocity in figures $1 \mathrm{a}$ and $1 \mathrm{~b}$ correspondingly. The wave head runs with the sound speed $c_{0}$ into the gas and involves the particles into the motion. The wave tail is the gasvacuum interface propagating into the vacuum with the maximal achievable velocity $v$ 


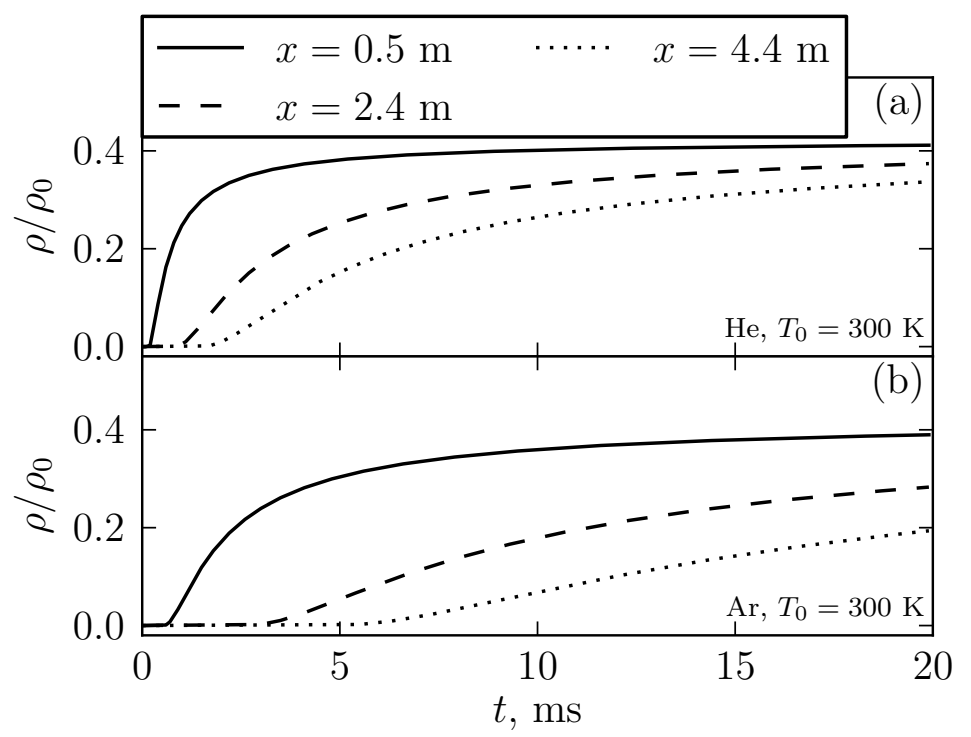

Figure 2. Flattening of the density pulse along the delivery tube for the rarefaction wave in $\mathrm{He}(\mathrm{a})$ and $\mathrm{Ar}(\mathrm{b})$.

of $2 \cdot c_{0} /(\gamma-1)$. Inside the wave the solution is self-similar, i.e. it depends only on the ratio $x / t$. It is convenient to introduce a dimensionless variable $\xi \equiv x /\left(c_{0} t\right)$ and a parameter $n=2 /(\gamma-1)$, which is actually the internal degree of freedom, to write in the wave $(-1<\xi<n)$ :

$$
\begin{aligned}
& \bar{v} \equiv \frac{v}{c_{0}}=\frac{n \cdot(1+\xi)}{n+1} \\
& \bar{\rho} \equiv \frac{\rho}{\rho_{0}}=\left(\frac{n-\xi}{n+1}\right)^{n}
\end{aligned}
$$

As obvious from the solution, gas particles of different density have also different velocities. It results in flattening of the gas front as it propagates along the $x$-axis. It is illustrated in figure 2, where density waveforms for Ar and He are plotted for three locations approximately corresponding to those used later for calibration. Density growth rate changes considerably between the shown locations, the front becomes shallower for larger distances. The flattening depends on the gas mass: for a heavier gas the front is smoothed stronger than for a lighter one.

\subsection{Number of particles delivered until time $t$.}

By using the given solution it is possible to calculate the number of particles $N(x, t)$ delivered beyond a point $x$ until time $t$. Aiming at a later comparison with the fuelling efficiency we normalize the value to the number of particles stored initially in the valve volume $V: \bar{N}(x, t) \equiv N(t) /\left(\rho_{0} \cdot V\right)$. For a fixed point $x$, integration in time can be 


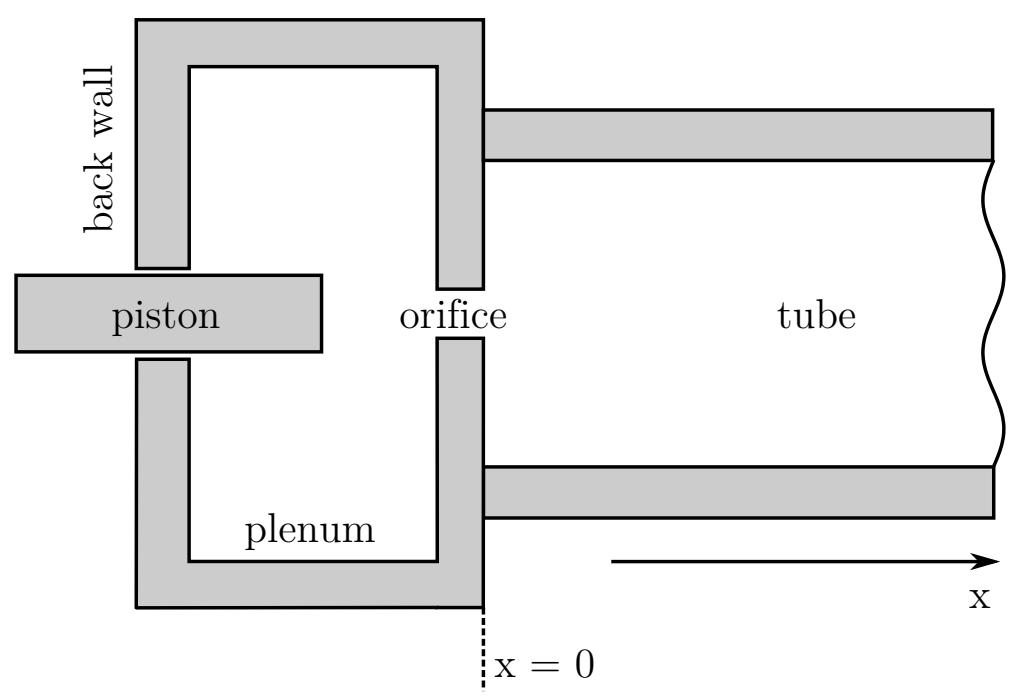

Figure 3. A schematic view of the valve orifice. The piston is drawn to the left to open the gas plenum through the orifice into the delivery tube. The tube is much longer than a typical valve size. The shown ratio between orifice and tube diameters is chosen only to illustrate the problem.

changed to the dimensionless variable $\xi$ :

$$
\bar{N}(x, t)=\frac{A \cdot K \cdot x}{V} \cdot \int_{\xi(t)}^{n} \bar{\rho}(\xi) \bar{v}(\xi) \frac{d \xi}{\xi^{2}}
$$

where $A$ is the cross-sectional area of the pipe and $K$ is a correction factor to be discussed later. By using equations 6, applying the binomial theorem and rearranging the series the integral can be easily taken:

$$
\bar{N}(t)=\frac{A \cdot K \cdot x}{V} \cdot \frac{n^{n}}{(n+1)^{n+1}} \cdot \sum_{k=0}^{n+1} \frac{(-1)^{k-1} \cdot(n+1) !}{(n-k+1) ! \cdot k !} \cdot\left(1-\left(\frac{\xi}{n}\right)^{k-1}\right)
$$

For the case of a mono-atomic gas $(\gamma=5 / 3 \Rightarrow n=3)$ the expression takes the form:

$$
\bar{N}(t)=\frac{A \cdot K \cdot x}{V} \cdot \frac{27}{256} \cdot\left(-4+\frac{3}{\xi}+2 \cdot \xi-\frac{4}{9} \xi^{2}+\frac{\xi^{3}}{27}\right)
$$

\subsection{Real flow in an MGI delivery tube and the ideal model.}

The real gas flow is more complicated than the proposed model: the flow is twodimensional at least close to the orifice and it is influenced by the finite size of the valve and the piston dynamics. In this section these limitations are briefly addressed. In general, the one-dimensional model can be applied to the flow in the delivery tube at sufficiently large distances. In figure 3 direction of the model $x$-axis is indicated, the beginning of the axis is chosen to be at the beginning of the tube. The latter choice is not critical in practice, since all measurement positions discussed here are at least 500 $\mathrm{mm}$ away from the orifice, while a typical wall thickness is about $10 \mathrm{~mm}$. 
The gas reservoir is of a limited extent and is nonuniform because of a piston (figure 3). The head of the rarefaction wave is reflected back and forth inside the valve in a very short time of the order of $0.1 \mathrm{~ms}$. Consequently, steady state parameters set in quickly close to the orifice. But at a large distance $x$ the flow remains in the unsteady regime for a longer time: the further away the observation point is, the longer the unsteady solution is valid. Only for such distant points $x>>d$, where $d$ is a typical valve size, the ideal model can be applied for a sufficiently long time before a steady state is established or a rarefaction wave induced by closing the valve comes.

The flow structure can be conveniently illustrated with the aid of an $x$-diagram (figure 4). The tail of the rarefaction wave, which is marked as "unsteady flow" in the figure, runs with the constant velocity into the vacuum. Behind it steady state parameters are transfered along the line "steady". Closing of the valve at time $t_{c l}$ produces a closing rarefaction wave propagating also to the right. At a certain position $x_{c l}$ the closing wave surpasses the steady state front and no steady state is possible afterwards. It is important that no disturbance can overtake the unsteady front, for it runs with the maximal achievable velocity and the sound speed tends to 0 at the front. Hence, there always exists a region, where the ideal model is valid. The duration of this unsteady region has to be assessed experimentally. It is worth noting that this flow structure is rather similar to that discussed for pulsed beams [29] and for a theory dealing with vacuum leaks [30].

The valve orifice is usually smaller than the tube cross-section (figure 3), so that a correction parameter $K$ describing the expansion close to the orifice has to be brought into our model (see equation 7). Because the steady state sets in rapidly at the orifice, the correction factor is likely to depend only on the ratio of the orifice area to the tube area $S_{o} / S_{t}$. At the same time, a velocity change caused by the expansion is assumed to be negligible. The validity of the latter assumption and the factor $K$ are to be determined during the calibration.

An additional deviation from the ideal model can be caused by not instantaneous opening of the piston, turbulence of the flow, condensation, viscosity and by velocity slip in the case of a gas mixture. These points are not addressed in this work. Instead, we again rely on the experimental confirmation of the model.

\section{Laboratory calibration of the flow model}

The flow model is validated by interferometric measurements of the gas density. The density was previously measured at three locations along the delivery tube $[13,14]$. The experiments were performed for the valves with an orifice diameter of 10, 18 and $28 \mathrm{~mm}$ and for the delivery tubes of an inner diameter of 38 and $102 \mathrm{~mm}$. The 10 and $18 \mathrm{~mm}$ valves were calibrated with the $38 \mathrm{~mm}$ tube, while the $28 \mathrm{~mm}$ valve was used with both 


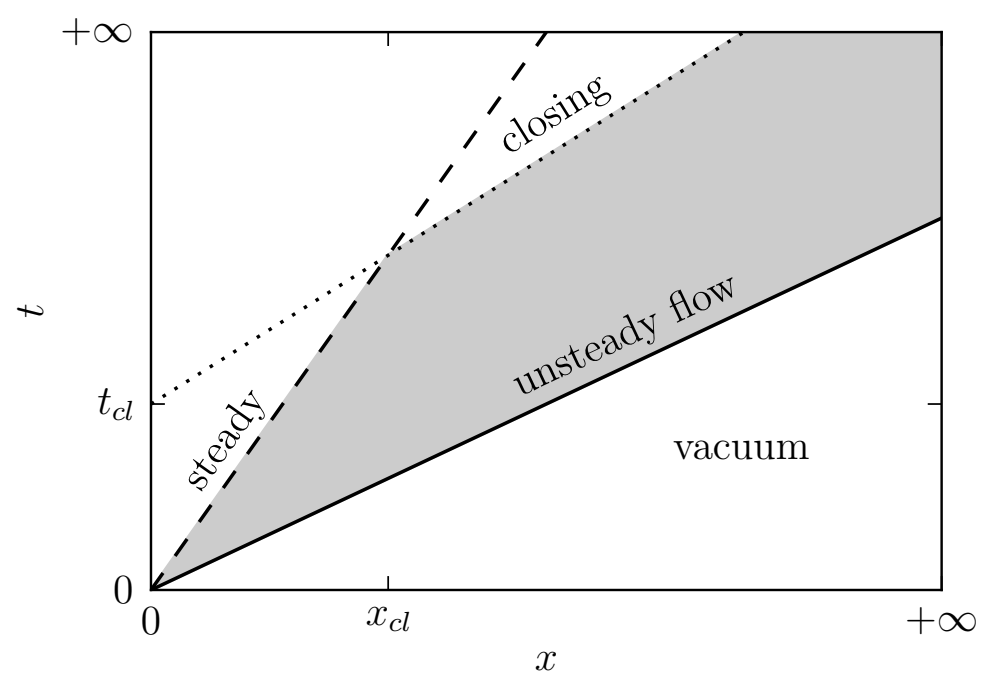

Figure 4. An $x t$-diagram of the flow in a delivery tube. The tail of the rarefaction wave is marked as "unsteady flow". The steady state front is marked as "steady". The closing rarefaction wave is marked as "closing". The valve orifice is located at $x=0$. The valve is closed at $t_{c l}$, and after the point $x_{c l}$ no steady state is possible. The region, where the ideal model is valid, is highlighted. The closing and steady state fronts are drawn schematically.

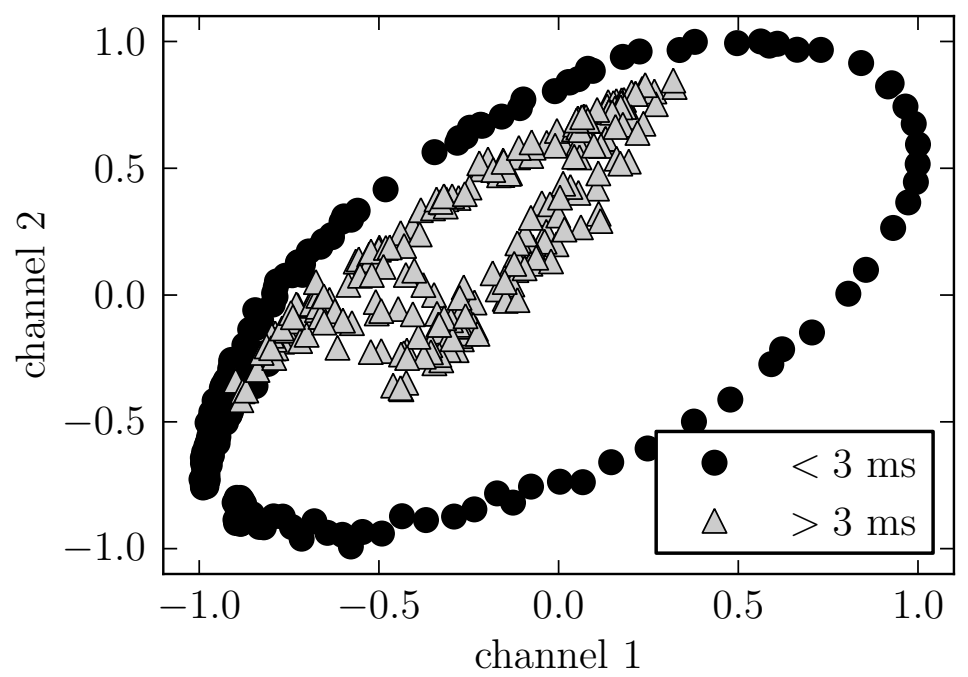

Figure 5. A Lissajous diagram of the two interferometer channels (10 bar He, 10 $\mathrm{mm}$ valve, $x=0.6 \mathrm{~m}$ ). Before $3 \mathrm{~ms}$ (circles) a clear ellipse can be seen. After $3 \mathrm{~ms}$ (triangles) there is a jump in the phase difference, that makes subsequent measurements impossible.

tubes. 


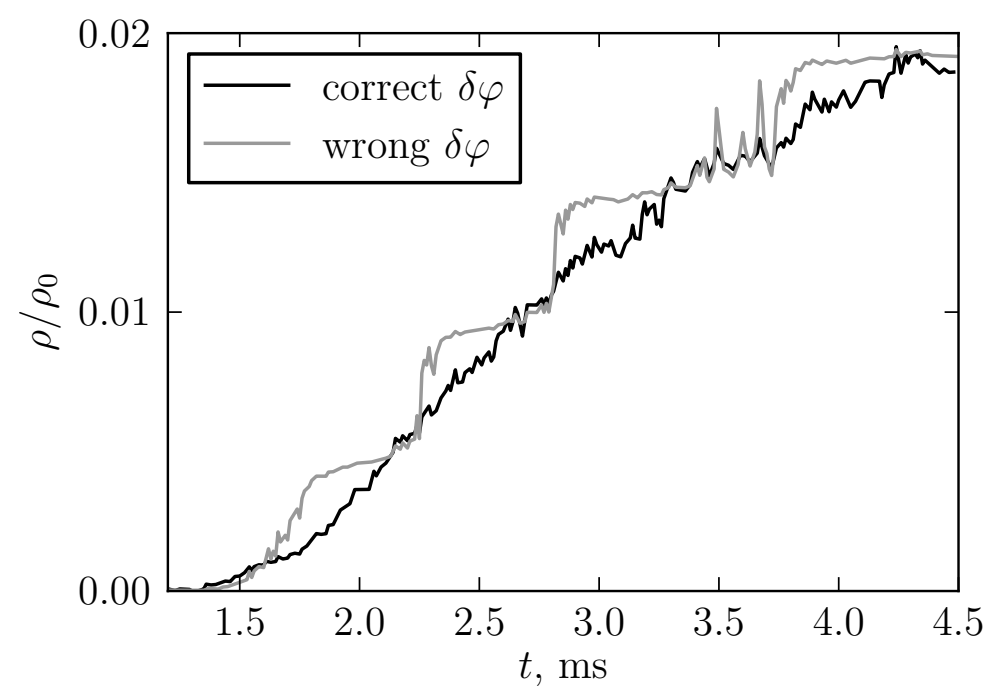

Figure 6. Importance of the correct phase difference $\delta \varphi$. If $\delta \varphi$ is not chosen properly, the density front form becomes erroneous (gray curve), even though the density does show a proper trend.

For the tests of the $28 \mathrm{~mm}$ valve a commercially available interferometer was employed, whereas for the 10 and $18 \mathrm{~mm}$ valves a self built interferometer was used. In the latter case the processing procedure has recently been significantly improved with respect to $[13,14]$. Since the improvements result in a noticeable change of the pulse form, first a short reanalysis of the data for the 10 and $18 \mathrm{~mm}$ valves is presented below. We also note that the commercial interferometer failed to record the data reliably at the closest to the valve location, because of a too steep front.

\subsection{Interferometric measurements of the gas density.}

On passing through a medium a light wave of wavelength $\lambda$ accumulates a phase shift:

$$
\Delta \varphi=\frac{2 \pi K_{G D}}{\lambda} \int n d l=\frac{2 \pi K_{G D}}{\lambda} n L
$$

where $K_{G D}$ is Gladstone-Dale constant, $L$ is the traversed path and the density $n$ is assumed to be uniform. To distinguish the direction of the density evolution, i.e. increase or decrease, in [13] the phase change $\Delta \varphi$ was independently measured by two interferometer channels separated by a phase difference $\delta \varphi$. Let the signals from the two channels be $y_{1}$ and $y_{2}$, then:

$$
\tan (\Delta \varphi)=\cot (\delta \varphi)-\frac{y_{2}}{y_{1}} \cdot \frac{1}{\sin (\delta \varphi)}
$$

Here two changes of the previous analysis are addressed. Firstly, the data set is restricted to a subset, where no irregularities like $\delta \varphi$ jumps are observed. And secondly, 
a correct choice of $\delta \varphi$ is shown to be important to recover the density reliably.

It is convenient to plot the data from both interferometer channels in the plane $\left(y_{1}, y_{2}\right)$. For a fixed phase difference $\delta \varphi$ such a plot is an ellipse, as shown in figure 5 for $t \leq 3 \mathrm{~ms}$. In some experiments abrupt changes of $\delta \varphi$ resulting in a deviation from the ellipse are observed, figure 5 for $t>3 \mathrm{~ms}$. Such cases as well as cases, where a fringe jump is found, are omitted from the present analysis, because a correct front form can not be guarantied. Possible reasons for the jumps include turbulence, gas condensation and tube oscillations. A further discussion of these phenomena is beyond the scope of the paper.

The phase difference $\delta \varphi$ is a crucial parameter for recovering the density. If a wrong value is assumed, the reconstructed density exhibits steps and the form becomes erroneous, even though it does show a proper trend, as illustrated in figure 6 . Here, the correct difference was found from intersections of the ellipse (figure 5) with the axes, which are equal to sine of $\delta \varphi$. In the previous work [13] the difference was assumed to be always 90 degrees. Since a readjustment of the setup was not performed between shots and especially after moving the system to a different position, this assumption does not hold for all cases. This is especially obvious from the Lissajous diagrams in the plain $\left(y_{1}, y_{2}\right)$ being not circles. Therefore, the raw data from [13] were reanalyzed for the whole reliable subset defined above.

\subsection{Comparison of the flow model to the interferometric measurements.}

The ideal model (equation 6) predicts that the normalized density waveform does not depend on the initial density $\rho_{0}$. It was indeed confirmed by the interferometric measurements for all used conditions. In figure 7 one finds an example of the normalized densities $\rho / \rho_{0}$ at one of locations for different initial pressures in the valve. The density fronts coincide to a good accuracy, a small difference is observed only at the falling part of the curves. The falling part is likely to be determined by closing of the valve, that strongly depends on the initial pressure [24]. The late phase being not relevant for the present analysis, the density waveform for a particular location, valve and gas was averaged over all available pressures to minimize noise.

It is natural to compare the flow model and the measurements in dimensionless variables $\rho / \rho_{0}$ and $\xi=x /\left(c_{0} \cdot t\right)$. In figure 8 , the Ne measurements at all used tube locations $x$ are plotted in these coordinates. Figure 8a presents the results for the $10 \mathrm{~mm}$ valve, and figure $8 \mathrm{~b}$ does the same for the $28 \mathrm{~mm}$ one. The experimental densities were averaged over the initial pressure, as explained above. For the $28 \mathrm{~mm}$ valve the data from the first measurement position are not available. For the $10 \mathrm{~mm}$ valve the time axis was corrected by a typical delay of $0.25 \mathrm{~ms}$ found earlier in observing the piston 


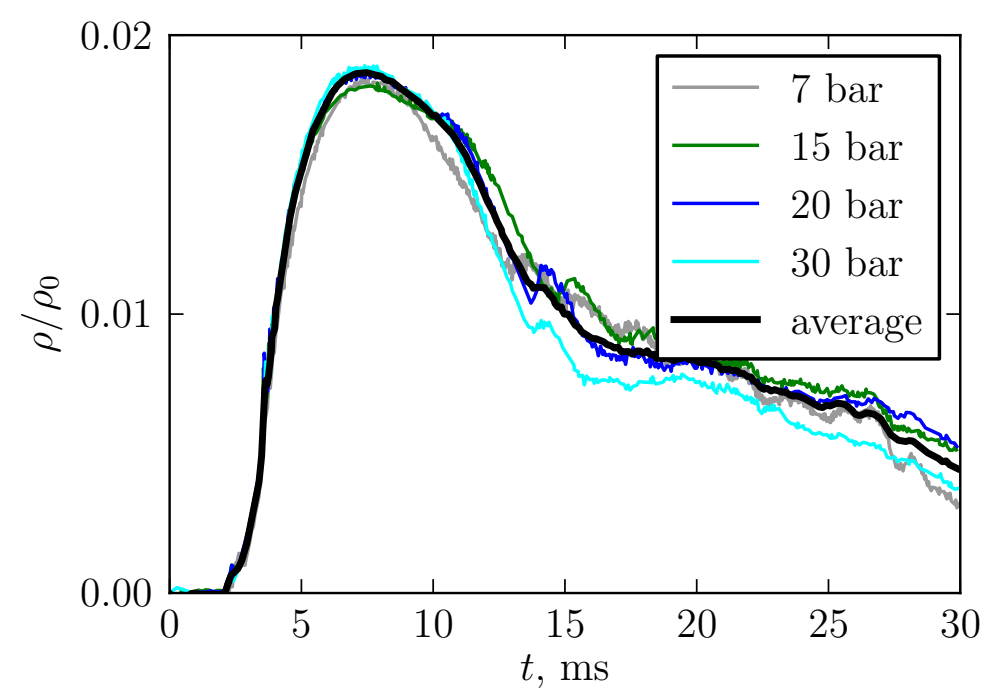

Figure 7. Normalized He density measured $4.5 \mathrm{~m}$ away from the $10 \mathrm{~mm}$ valve. The form of the density front is independent on the initial gas pressure.

motion [24]. The rising density front, that is in fact falling with respect to $\xi$, can be fitted with the flow model by choosing the correction factor $K$ of 0.1 for the $10 \mathrm{~mm}$ case and 0.6 for the $28 \mathrm{~mm}$ one. The accuracy of the fitted model is better than $30 \%$. The model remains valid as long as $\xi$ stays above about $0.6-0.75$, with the exact value depending on the valve, tube configuration and the measurement location. For the TEXTOR setup $(x=1.5 \mathrm{~m})$ this limits the time to be smaller than $2.5 \mathrm{~ms}$ for He, $5 \mathrm{~ms}$ for $\mathrm{Ne}$ and $7 \mathrm{~ms}$ for Ar, which is almost two times longer than a typical disruption time.

It is worth noting that a small steady state is observed only in some configurations close to the valve orifice, which is in full agreement with the $x$-diagram in figure 4 . If the steady state is present, the velocity during this phase is below the sound speed $c_{0}$.

The correction factor $K$ does not vary with the gas, but depends only on the ratio of the output orifice area $S_{o}$ to the tube area $S_{t}$, figure 9 . Combination of the $28 \mathrm{~mm}$ valve and the $102 \mathrm{~mm}$ tube leads to almost the same value as the $10 \mathrm{~mm}$ valve and the $38 \mathrm{~mm}$ tube. During the calibration the 10 and $18 \mathrm{~mm}$ valves were operated under conditions, where a full piston stroke was not achieved. In these two cases the output surface limiting the flow is given by a cylinder projected by the piston stroke: $S_{o}=\pi d h$, where $d$ is the geometrical orifice diameter and $h$ is the stroke measured with the aid of a fast camera [24]. The best linear fit to the experimental data is given by:

$$
K=1.05 \cdot \frac{S_{o}}{S_{t}}+0.03
$$

To sum up, the ideal flow model (equation 6) was demonstrated to describe the 


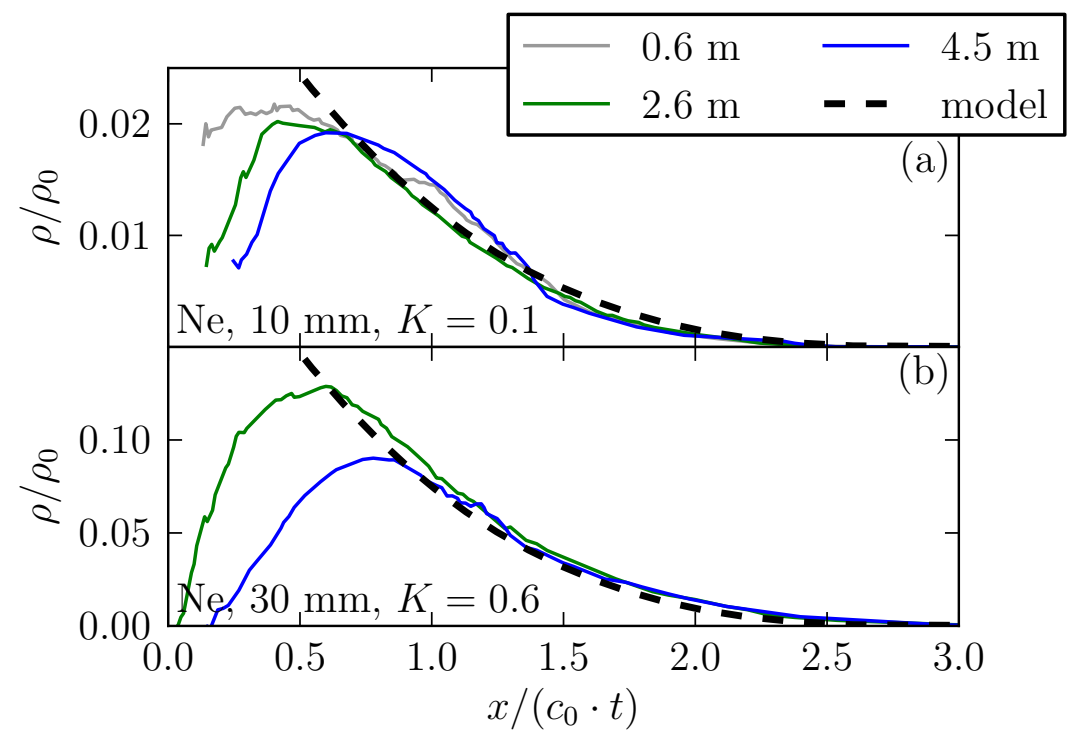

Figure 8. Comparison of the flow model to the experimental data for Ne. (a) - $10 \mathrm{~mm}$ valve. (b) - $30 \mathrm{~mm}$ valve. The experimental densities were averaged over the initial pressure in the valve. The model describes the experiment with a good accuracy for $x /\left(c_{0} \cdot t\right)$ larger than about $0.6-0.75$.

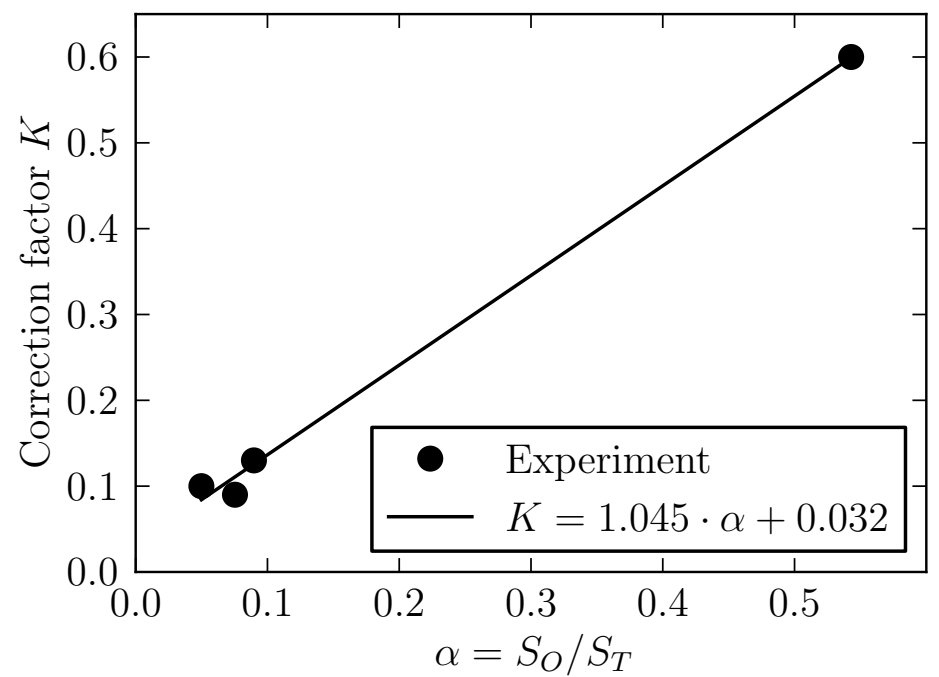

Figure 9. Scaling of the model correction factor $K$ with the ratio of the valve orifice area to the area of the delivery tube.

real flow with an accuracy of about 30\%. The model is valid for times and distances that satisfy the inequality $x /\left(c_{0} t\right)>0.6$. The model correction factor can be found from equation 12. This allows us to use equations 8 and 9 to estimate the number of delivered particles in tokamak experiments on massive gas injection. 


\section{Massive gas injection at TEXTOR}

In this section the fuelling efficiency of massive gas injection in TEXTOR is discussed. First the experimental procedure and the method of measuring the fuelling efficiency are introduced. Which is followed by a comparison of the experimental results with the gas flow model.

At TEXTOR an $18 \mathrm{~mm}$ fast valve for massive gas injection is mounted on the top of the machine, approximately above the plasma center. The valve volume is $50 \mathrm{ml}$, which can be filled with gas in the pressure range $1-20$ bar. A variation of the initial pressure corresponds to a change of the impurity density to be expected in the plasma $n_{\text {ideal }}=N / V$, where $N$ is the number of atoms in the valve and $V=17 \mathrm{~m}^{3}$ is the volume of the TEXTOR vacuum vessel. The valve is connected to TEXTOR through a $1.5 \mathrm{~m}$ long $38 \mathrm{~mm}$ inner diameter delivery tube. It is to be mentioned that earlier the delivery system was slightly different: the connection tube was about $0.9 \mathrm{~m}$ long and the rest the gas travelled freely (partially limited to a $120 \mathrm{~mm}$ diameter) inside the vessel. Because the difference in results between these two cases is inside the errors of our method, we do not distinguish them. The system was triggered preemptively in the flat top of stable discharges. The following gasses were used: He, Ne, Ar, Kr, Xe and a mixture of 10\% of Ar with deuterium. During the gas/pressure scan the plasma parameters were kept the same: toroidal field $B_{t}=2.25-2.4 \mathrm{~T}$, plasma current $I_{p}=300-350 \mathrm{kA}$, electron density $\left\langle n_{e}\right\rangle=2 \cdot 10^{19} \mathrm{~m}^{-3}$ and thermal energy $E_{t h} \approx 40 \mathrm{~kJ}$.

A typical phenomenology of the experiments is illustrated with an example of a low pressure argon injection in figure 10. In this figure the time is given relative to the valve trigger. Having travelled through the delivery tube the gas first cools the plasma edge, while the core remains hot (figure 10a). After some time the cold front reaches the $q=2$ surface $[12,31]$ and the electron temperature falls simultaneously at the $q=2$ surface and in the plasma centre. At the same time a burst of magnetic oscillation is seen on Mirnov coils (figure 10b): the thermal quench takes place. Later the plasma current decays on a resistive time scale (figure 10c), at this time the electron temperature is of the order of $10 \mathrm{eV}$ only. This last stage is known as current quench (CQ). The start of the current quench is defined here as the maximum of the current in the transient current spike.

In the case of relatively low pressure injections ( $p_{0} \leq 15$ bar) of $\mathrm{Ar}, \mathrm{Kr}$ and $\mathrm{Xe}$ a runaway plateau appears in the current quench, as illustrated by example in figure 10 . At about $7 \mathrm{~ms}$ the current deviates from a smooth decay and preserves a high value. No special settings are prepared for the plasma control system in MGI experiments, therefore in the current quench the external loop voltage is raised to its maximum. The electric field applied in such a way by the external transformer is below about $1 \mathrm{~V} / \mathrm{m}$ and is ramped up on a millisecond time scale, so that it does not influence the runaway generation strongly. Later, after $40 \mathrm{~ms}$ in the shown example, the plateau is terminated 


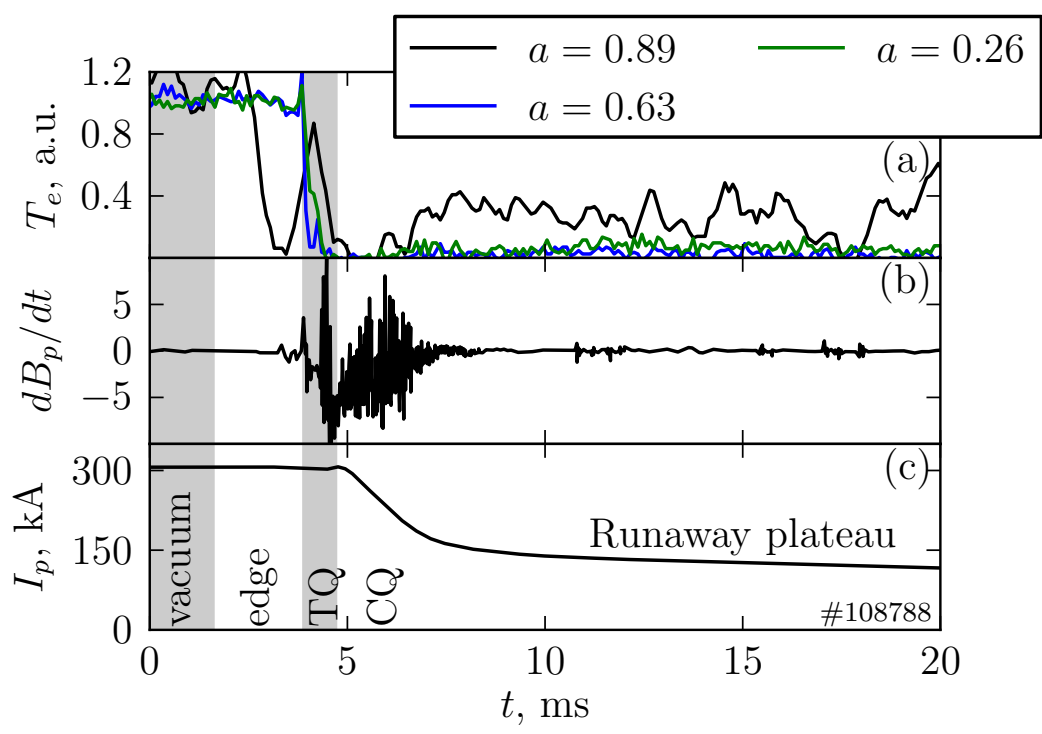

Figure 10. Phenomenology of the massive gas injection. An example of a low-pressure argon injection. (a) - normalized electron temperature at the plasma edge, at the $q=2$ flux surface and in the plasma centre. (b) - Mirnov coil signal. (c) - plasma current. Stages of the experiment are: "vacuum" - vacuum flow from the valve to the plasma; "edge" - cooling of the plasma edge; "TQ" - thermal quench; "CQ" - current quench. The time axis is given relative to the valve trigger.

to the wall because of a poor horizontal position control. The horizontal position is adjusted from electron density measurements along several chords, which is not adequate for the runaway beam. For the runaway suppression the impurity density in the first phase of the current quench, i.e. the fuelling efficiency, is of great importance. A further discussion of the detection of runaways and of dependence of the runaway population on the number of injected particles can be found in [12].

\subsection{Measurements of the fuelling efficiency}

The fuelling efficiency is defined as the ratio of the density of impurities $n_{Z}$ in the plasma at the current quench to the density one can expect for the number of particles $N$ stored initially in the valve plenum (figure 3 ):

$$
\mathcal{E} \equiv \frac{n_{Z}}{N / V}
$$

Where $V$ is the TEXTOR vacuum vessel volume. To find the efficiency a way of measuring the impurity density is required. Here, a zero-dimensional model of the current quench is used. The model is fitted to reproduce the measured current time traces. Another important comparison between the model and the experiment could be the electron density. Unfortunately, the electron density diagnostic at TEXTOR fails during the massive gas injection experiments. 


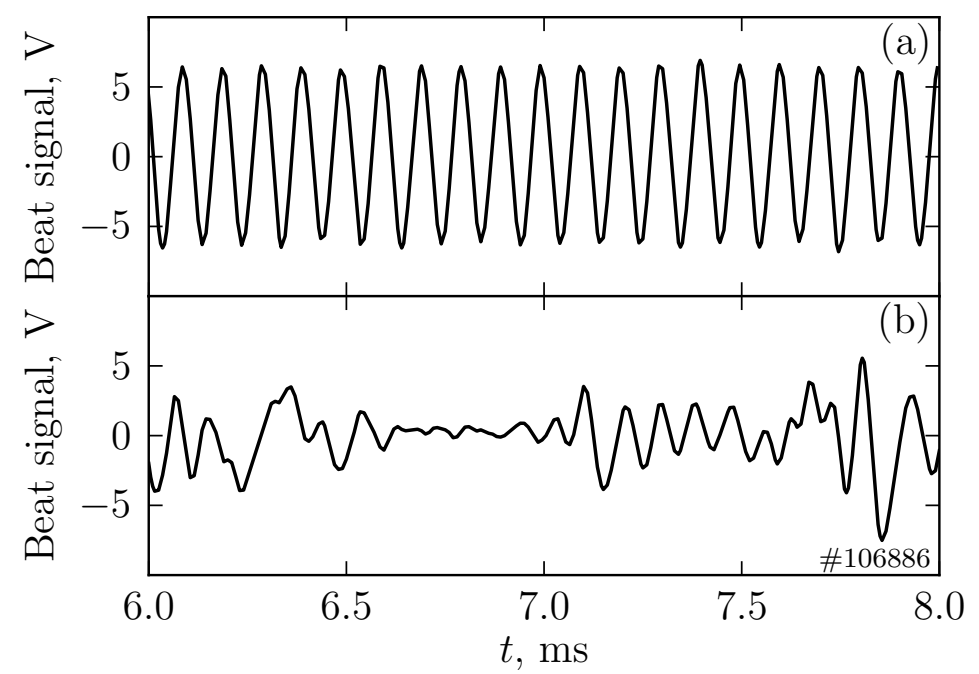

Figure 11. Beat signal of the HCN interferometer. (a) - flat top of a stable discharge, regular oscillations with the modulation frequency are observed. (b) - during massive gas injection the amplitude of the probing beam drops. As a result, the beat signal is small and very irregular, so that no density measurements are possible in this case.

The standard technique of the electron density measurement at TEXTOR is an $\mathrm{HCN}$ interferometer. The interferometer is of a phase-modulated Mach-Zehnder type [32]. The modulation is achieved according to Véron [33] by reflecting the reference beam from a rotating grating, which gives the frequency shift of $10 \mathrm{kHz}$. A phase shift due to passing of a probing beam through the plasma is detected than on the beat signal having the same frequency as the modulation one. An example of a normal beat signal is depicted in figure 11a for a steady state plasma. However, during the massive gas injection the laser beam is refracted on a large density gradient and does not reach the detector. As a result, the amplitude of the beat signal drops to zero or the signal has very strong irregularities (figure 11b), so that no reliable density measurements are possible from about the start of the thermal quench until very late in the current quench, when the current drops to a value below about $20 \mathrm{kA}$.

The used model of the current quench includes ohmic current decay $\left(L, I_{\Omega}, E\right)$, inductively coupled currents in the vessel $\left(L_{\mathrm{V}}, I_{\mathrm{V}}, \tau_{\mathrm{V}}\right)$, runaway generation $\left(n_{\mathrm{re}}, I_{\mathrm{re}}\right.$, $\left.L_{\text {re }}\right)$ and power balance:

$$
\begin{array}{ll}
\frac{d n_{\mathrm{re}}}{d t} & =f_{\mathrm{I}}+\left(\gamma_{\mathrm{II}}-\gamma_{\ell}\right) n_{\mathrm{re}} \\
\frac{d}{d t}\left(L I_{\Omega}+L_{\mathrm{re}} I_{\mathrm{re}}+L_{\mathrm{v}} I_{\mathrm{V}}\right) & =-2 \pi R_{0} E \\
\frac{d}{d t}\left(I_{\mathrm{V}}+I_{\Omega}+I_{\mathrm{re}}\right) & =-\frac{I_{\mathrm{V}}}{\tau_{\mathrm{V}}}
\end{array}
$$




$$
\eta \frac{I^{2}}{S^{2}}=n_{e} n_{Z} L_{Z}
$$

Where $f_{\mathrm{I}}, \gamma_{\mathrm{II}}$ and $\gamma_{\ell}$, are the primary, secondary generation and the loss rate of the runaway electrons respectively. $R_{0}$ is the major TEXTOR radius, $\eta$ is the Spitzer resistivity, $S$ is the plasma cross section, and $L_{Z}$ is the radiative loss rate. Further details of the model are given in [12]. Both the loss rate $L_{Z}$ and the ionization state of impurities are calculated in coronal equilibrium: ADAS [34], for argon also [35,36], for helium also [37] and for krypton also $[38,39]$. It is to be noted that there also exist more elaborated current quench models using state-resolved atomic physics [40]. The experimental current evolution was fitted by the least square method by choosing a proper value for $n_{Z}$. The averaged difference between the calculated current and the experimental one could be reduced to a few kiloampers.

The uncertainty associated with the model stem from model parameters being poorly known. From a sensitivity analysis [12] it follows that the most critical parameter is the plasma radius, i.e. the current density. In this work the plasma radius was varied in what we consider to be a physical range: the largest radius is given by the limiter position, while the smallest one is given by the condition that the current redistribution corresponds to a decrease of the internal inductivity $\ell_{i}$ at the transient current peak. The latter condition results in the smallest possible radius of about $0.3 \mathrm{~m}$. Visible camera observations indicate that the radius changes during the current quench. The final results presented here were averaged over the all possible cases. And the limits give the error bars. We estimate the mean error to be about a factor of two. Only for the discharges exhibiting a runaway plateau the spread can be larger owing to an exponential dependence of the runaway generation on the electric field. To reduce the uncertainties additional diagnostics in the current quench are desirable.

\subsection{Dependence of the fuelling efficiency on the gas mass}

To find a typical fuelling efficiency for a gas, the impurity density found as explained above was linearly fitted against the expected density $N / V$. The fuelling efficiency depends in fact also on the initial pressure, as illustrated later. Consequently, such a fit gives only a rough value, which is more typical for high pressure injections. The data set includes 4 discharges for He, 10 for the mixture of argon with deuterium, 9 for neon, 15 for argon, 6 for krypton and 9 for xenon. Dependence of the fuelling efficiency on the gas mass is summarized in figure $12 \mathrm{~b}$ with solid circles. The efficiency decreases significantly as the mass grows: the value descends from above 0.5 for He and about 0.2 for the argon-deuterium mixture to below 0.03 for $\mathrm{Kr}$ and Xe. The errors of the values are about a factor of 2 .

To estimate the relative number of particles $\bar{N}$ delivered to the plasma a typical time of the experiment has to be introduced. The beginning of the thermal quench 


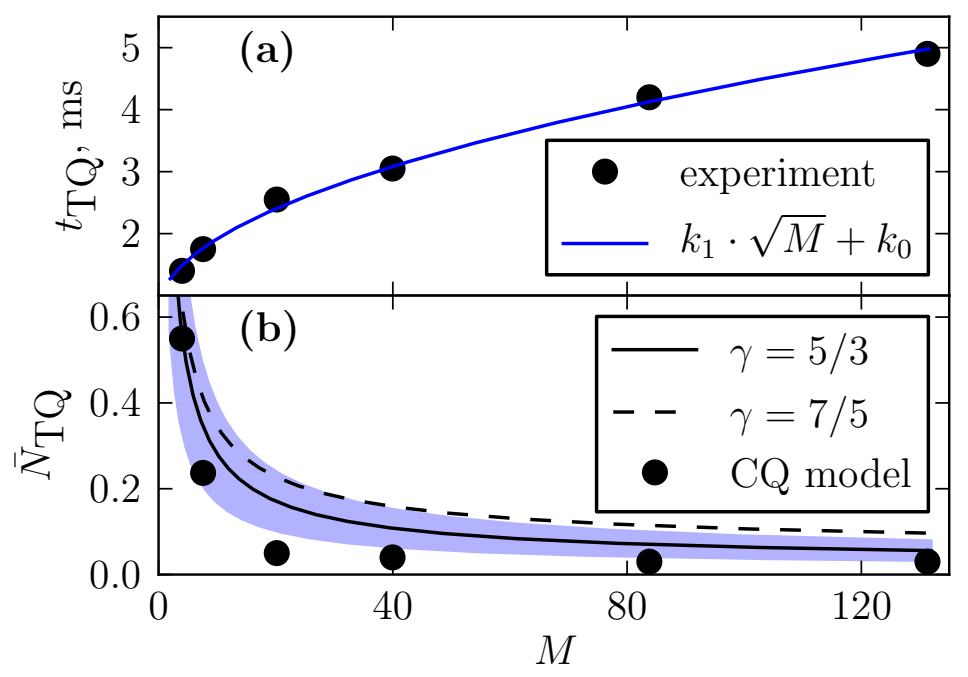

Figure 12. Dependence of the fuelling efficiency on the mass. (a) - thermal quench time. The solid line shows the fit used for the flow model. (b) - relative number of particles. The black circles stand for the results of the current quench modelling. The solid line is the number of particles delivered before the thermal quench for a monoatomic gas. The dashed line shows the same but for a diatomic gas. The filled area gives a typical uncertainty associated with the flow model for monoatomic gases.

was chosen as such a characteristic time, since only the atoms delivered before have a good chance to be redistributed over the plasma volume [22]. The start of the thermal quench was defined as an abrupt drop of the electron temperature at the $q=2$ flux surface. Variation of the thermal quench time averaged over the used pressures with the mass is presented in figure 12a. The experimental points can be fitted with a dependence of the form $t=k_{1} \cdot \sqrt{M}+k_{0}$, as shown in the figure with a solid line. A square root of mass scaling was assumed to take into account the sound speed change.

The dependence of the number of atoms delivered before the disruption on the mass is shown in figure $12 \mathrm{~b}$ with a solid line for mono-atomic gases and with a dashed line for diatomic gases. Here equations 8 and 9 were used with the correction factor for TEXTOR $K=0.25$ and the disruption time scaling from figure 12a. A color filled region in the figure indicates a typical uncertainty associated with the flow model for mono-atomic gases. It is to be noted that the experimental point $M=7.6$ corresponds to the mixture of $10 \%$ of argon with deuterium, i.e. it is better represented by the diatomic case. The flow model reproduces the trend found from the current quench model: the efficiency of fuelling decreases with the mass. To put it another way, the drop of the efficiency is due to the fall of the number of delivered particles. The gas pulse is smeared out (figure 2) and a small fraction of atoms at its front causes the thermal quench before the bulk of atoms can reach the plasma. Because both the smearing and the cooling capability increase with the mass, it is increasingly difficult to deposit 


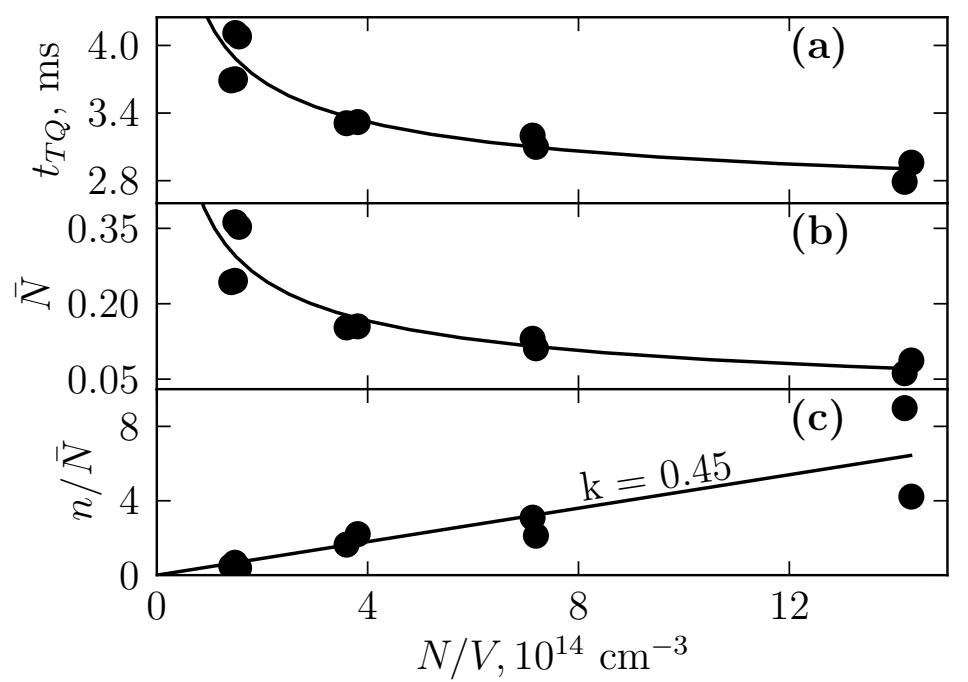

Figure 13. Scan of the initial pressure for argon. The pressure is given as the expected density $N / V$. (a) - thermal quench time; (b) - relative number of delivered atoms; (c) density found from the current quench model normalized by the delivered fraction. The linear fit in (c) gives the mixing efficiency. In figures (a) and (b) the lines are given only to guide the eye.

a large number of high- $Z$ atoms.

\subsection{Mixing efficiency. Pressure dependence of the fuelling efficiency.}

The injected density at the beginning of the current quench can be considered as a combination of two processes: (i) gas delivery before the thermal quench; and (ii) mixing or redistribution of the delivered atoms during the thermal quench. If the mixing is denoted as $\mathcal{M}$, the full efficiency $\mathcal{E}$ can be represented as:

$$
\begin{aligned}
\mathcal{E} & =\mathcal{M} \cdot \bar{N}\left(t_{T Q}, L, c_{0}\right) \\
t_{T Q} & =f(\rho v, \cdots)
\end{aligned}
$$

Where the delivered fraction $\bar{N}$ depends on the disruption time $t_{T Q}$, distance to the valve $L$ and the sound speed $c_{0}$. The disruption time $t_{T Q}$ itself depends on the flow rate $\rho v$ and plasma parameters. Given the fact that the thermal quench duration does not vary strongly with the injection parameters, the mixing $\mathcal{M}$ can be assumed to be constant.

It could have been possible to find the mixing $\mathcal{M}$ directly from the mass dependence of the fuelling efficiency. However, we illustrate a more precise method where every experimental point $n_{Z}$ is normed by the fraction $\bar{N}$ and this data set is than fitted against the expected density $N / V$. At the same time a change of the fuelling efficiency 
with the initial pressure is illustrated.

In figure 13 one finds a scan of the initial gas pressure for argon. The initial pressure is given as the expected density. As the pressure is increased the pre-disruptive phase shortens (figure 13a), which leads to a reduction of the delivered fraction, and consequently of the fuelling efficiency. The delivered fraction descends from about 0.3 for the low pressure puffs to about 0.06 for the high pressure ones, figure $13 \mathrm{~b}$. The impurity density normalized by the delivered fraction $n_{Z} / \bar{N}$ can be linearly fitted against the expected density with a good accuracy, figure 13c. The best fit in the shown case is achieved with the mixing of 0.45 .

The mixing efficiency turned out to have no clear dependence on the gas mass and to scatter in the range from 0.3 to 0.8 with an average value of about 0.5 . The highest value of 0.8 is found for He, where the accuracy of finding $n_{Z}$ is the lowest due to a possible influence of intrinsic impurities. The particles not mixed during the thermal quench continue to slowly diffuse into the plasma in current quench, but at least for our conditions they do not seem to play an important role in the process dynamics.

\section{Discussion and conclusions}

For collisional suppression of runaway electrons in ITER disruptions the total electron density must be rapidly increased by several orders of magnitude. To design a suitable massive gas injection system it is important to know efficiency of the method. A uniform definition of the fuelling efficiency was lacking. We accept the definition used at JET, according to which the efficiency is given as the ratio of the number of atoms at the current quench, that is at the time when the suppression is to take place, to the number of atoms stored initially in the injector. The efficiency is composed of two terms: fraction of atoms delivered before the thermal quench and the mixing efficiency of these atoms into the plasma core. Importance of these two factors was analyzed in this work.

To estimate the number of particles delivered to the plasma, a gas flow model was developed. This model is based on one-dimensional unsteady expansion of gas into vacuum and includes an additional correction factor describing gas expansion close to the injector orifice.

The flow model was validated with available measurements of a TEXTOR-like injection system $[13,14]$. At the same time the calibration set from [13] was improved due to a more suitable processing The model correction factor was found to depend on the ratio of the orifice area to the delivery tube area. The correction factor for TEXTOR was determined to be $0.25 \pm 0.07$. The model can be applied as long as $x /\left(c_{0} t\right)$ remains 
larger than 0.6. It is worth noting that the injection system used at TEXTOR is of the same type as the one at JET and therefore the same model could be also applied there with the correction factor of about 0.10 .

The fuelling efficiency of massive gas injection at TEXTOR was determined uniformly for the following gases: $\mathrm{He}, \mathrm{Ne}, \mathrm{Ar}, \mathrm{Kr}, \mathrm{Xe}$ and mixture of $10 \%$ of Ar with deuterium. It was shown that the efficiency drops from above 0.5 for He to below 0.03 for $\mathrm{Kr}$ and Xe. From the flow model it follows that this tendency is due to a decrease of the number of delivered atoms: the gas pulse is smeared out and a fraction of atoms at its front causes the thermal quench before the majority of the released atoms can reach the plasma. Because both the smearing and the cooling capability increase with the gas mass, it is increasingly difficult to deposit a large number of high- $Z$ atoms. It was also shown that the fuelling efficiency decreases as the initial pressure in the valve is increased, which is due to a shortening of the pre-disruptive phase.

The efficiency of mixing delivered atoms into the plasma during thermal quench was found to be about 0.5 for all gases used at TEXTOR. This value seems to be higher than 0.2 typically found in DIII-D [22]. However, here the efficiency was normalized to the number of atoms delivered before the thermal quench, in contrast to DIII-D, where the atoms delivered up to the middle of the current quench are considered. If our value is normalized instead to the number of particles delivered before the current quench, the mixing efficiency is to be reduced by a factor of about 2, which would make our result consistent with that of DIII-D. A comparison with the results from ASDEX Upgrade [5] is not easily possible, since their values are not normalized to the delivered particles and contain a mean charge state. A more thorough inter-machine study is certainly needed.

The most important factor determining the full fuelling efficiency is the number of atoms delivered before the thermal quench. Since this number depends on the duration of the pre-disruptive phase, to predict the efficiency for ITER a reliable inter machine scaling of the disruption time is required. To our opinion, it is such a scaling that will determine whether massive gas injection can succeed in preventing the runaway electrons. It is important to mention that such a scaling should depend on plasma parameters, in particular on the edge safety factor: the smaller the edge safety factor is, the earlier the disruption is to be expected, and consequently a lower efficiency can be achieved. In establishing the scaling one has to take care to account for different flow rates in different experiments.

The following two conclusions concerning massive gas injection in ITER can also be drawn from our results: (i) the distance between the plasma and the valve is an important parameter, and (ii) because the delivered fraction for medium- and high- $Z$ gases is low, the number of particles should be increased by enlarging the gas flow rate, i.e. the gas pressure, the tube cross section, the number of mitigation valves used. For 
a quantitative description of these factors a scaling of the disruption time is missing. However, the first proposal can be illustrated, for example, by the experience of ASDEX Upgrade [5], where shortening of the valve-plasma distance from $1.5 \mathrm{~m}$ to $10 \mathrm{~cm}$ improved the fuelling efficiency by a factor of $2-3$.

Another important question is the choice of an optimum gas. Because of the presence of the bound electrons in the condition for the runaway suppression, high- $Z$ gases seem to be preferable. The use of high- $Z$ gases would minimize loads on the vacuum and tritium-processing systems. On the other hand the fuelling efficiency decreases with $Z$. The TEXTOR results hint that high- $Z$ gases can still be advantageous: the same total electron density can be achieved with $1.5-2$ times lower pressure. However, this can be a coincidence for a particular valve-plasma distance and plasma parameters. A further study in this direction can also be helpful for the ITER design.

\section{References}

[1] Shimada M et al. Progress in the ITER Physics Basis Chapter 1: Overview and summary 2007 Nuclear Fusion 47 S1-S17

[2] Sugihara M, Campbell D J, Gribov Y, Loarte A, Mukhovatov V, Pitts R A, Putvinski S and Shimada M 2009 Disruption and runaway electron mitigation on ITER 36th EPS Plasma Physics Conference, Sofia, June 29 - July 3, 2009 ECA Vol.33E, P-4.165 (2009)

[3] Whyte D and et al 2003 Journal of Nuclear Materials 313-316 1239-1246

[4] Granetz R, Whyte D, Izzo V A, Biewer T, Reinke M L, Terry J, Bader A, Bakhtiari M, Jernigan $\mathrm{T}$ and Wurden G 2006 Nuclear Fusion 46 1001-1008

[5] Pautasso G, Fuchs C J, Gruber O, Maggi C F, Maraschek M, Putterich T, Rohde V, Wittmann C, Wolfrum E, Cierpka P and Beck M 2007 Nuclear Fusion 47 900-913

[6] Pautasso G, Büchl K, Fuchs J C, Gruber O, Herrmann A, Lackner K, Lang P, Mast K, Ulrich M and Zohm H 1996 Nuclear Fusion 36 1291-1297

[7] Taylor P L, Kellman A G, Evans T E, Gray D S, Humphreys D A, Hyatt A W, Jernigan T C, Lee R L, Leuer J A, Luckhardt S C, Parks P B, Schaffer M J, Whyte D G and Zhang J 1999 Physics of Plasmas 6 1872-1879

[8] Yoshino R, Kondoh T, Neyatani Y, Itami K, Kawano Y and Isei N 1997 Plasma Physics and Controlled Fusion 39 313-332

[9] Baylor L, Combs S, Foust C, Jernigan T, Meitner S, Parks P, Caughman J, Fehling D, Maruyama S, Qualls A, Rasmussen D and Thomas C 2009 Nuclear Fusion 49085013 (8pp)

[10] Jernigan T C, Baylor L, Combs S, Commaux N, Meitner S, Hollmann E M, Yu J, Humphreys D A, Van Zeeland M and Wesley J 2009 Large, shattered pellets for disruption mitigation in DIII-D $51^{\text {st }}$ Annual APS Meeting of the Division of Plasma Physics, Atlanta, Georgia, USA, November 2-6 2009, JP8.90

[11] Hollmann E M, Izzo V, James A, Yu J H, Baylor L R, Commaux N, Jernigan T, E E T, Eidietis N, Humphreys D A, Parks P B, Strait E J, Van Zeeland M A, Wesley J C and Wu W 2009 Experiments in DIII-D toward achieving rapid shutdown with runaway electron suppression $51^{\text {st }}$ Annual APS Meeting of the Division of Plasma Physics, Atlanta, Georgia, USA, November 2-6 2009, XI3.4

[12] Bozhenkov S A, Lehnen M, Finken K H, Jakubowski M W, Wolf R C, Jaspers R, Kantor M, Marchuk O V, Uzgel E, van Wassenhove G, Zimmermann O and Reiter D 2008 Plasma Physics and Controled Fusion 50105007 (18pp) 
[13] Finken K H, Lehnen M and Bozhenkov S A 2008 Nuclear Fusion 48115001 (10pp)

[14] Finken K H, Lehnen M and Bozhenkov S A 2011 Nuclear Fusion 51033007 (12pp)

[15] ITER Physics Expert Group on Disruptions, Plasma Control, and MHD and ITER Physics Basis Editors, 1999 Nuclear Fusion 392251 - 2389

[16] Hender T C et al. Progress in the ITER Physics Basis Chapter 3: MHD stability, operational limits and disruptions 2007 Nuclear Fusion 47 S128-S202 (beginning of chapter)

[17] Helander P, Eriksson L G and Andersson F 2002 Plasma Physics and Controlled Fusion 44 B247B262

[18] Knoepfel H and Spong D A 1979 Nuclear Fusion 19 785-829

[19] Lehnen M, Bozhenkov S A and Abdullaev S S 2008 Physical Review Letters 100255003 (4pp)

[20] Connor J and Hastie R 1975 Nuclear Fusion 15 415-424

[21] Fussmann G 1979 Nuclear Fusion 19 327-334

[22] Hollmann E M, Jernigan T C, Parks P B, Boedo J A, Evans T E, Groth M, Humphreys D A, James A N, Lanctot M J, Nishijima D, Rudakov D L, Scott H A, Strait E J, Van Zeeland M A, Wesley J C, West W P, Wu W and Yu J H 2008 Nuclear Fusion 48115007 (12pp)

[23] Lehnen M, Alonso A, G Arnoux and S B, Brezinsek S, Eich T, Finken K, A Huber and S J, Kruezi U, Morgan P, Plyusnin V, Reux C, Riccardo V, Sergienko G and Stamp M 2009 First experiments on massive gas injection at JET - consequences for disruption mitigation in JET and ITER 36th EPS Plasma Physics Conference, June 29 - July 3, 2009, Sofia, Bulgaria, O2.001

[24] Bozhenkov S A, Finken K H, Lehnen M and Wolf R C 2007 Review of Scientific Instruments 78 033503 (7pp.)

[25] Landau L D and Lifshitz E M 1986 Hydrodynamics (Course of theoretical physics v. 6) (Moscow "Nauka")

[26] Stanyukovich K P 1971 Non-stationary fluid dynamics (in Russian) (Moscow "Nauka")

[27] Zel'dovich J B and Raizer Y P 1966 Physics of shock waves and high temperature phenomena (Moscow "Nauka")

[28] Courant R and Friedrichs K O 1948 Supersonic flow and shock waves (New York)

[29] Korobeishchikov N and Zarvin AE and Madirbaev V 2004 Technical Physics 49 973-981

[30] Takiya T, Higashino F, Terada Y and Komura A 1999 Journal of Vacuum Science and Technology 17 2059-2063

[31] Bozhenkov S A, Lehnen M, Finken K H, Jakubowski M W, Jaspers R, Wolf R C, Abdullaev S S, Kantor M, van Wassenhove G and Reiter D Runaway electrons after massive gas injections in TEXTOR: importance of the gas mixing and of the resonant magnetic perturbations. 35th EPS Plasma Physics Conference, 9-13 June 2008, Hersonissos, Crete, Greece, P-1.079

[32] Koslowski H R and Soltwisch H 1997 Fusion Engineering and Design 34-35 143-150

[33] Veron D, Certain J and Crenn J P 1977 Journal of the Optical Society of America 67 964-967

[34] Summers H 2001 The adas user manual, version 2.4 http://adas.phys.strath.ac.uk URL http://adas.phys.strath.ac.uk

[35] Fournier K B, Cohen M, May M J and Goldstein W H 1998 Atomic Data And Nuclear Data Tables $70231-254$

[36] Fournier K B, Cohen M, May M J and Goldstein W H 2000 Atomic Data And Nuclear Data Tables $74333-334$

[37] Post D E and Jensen R V 1977 Atomic Data And Nuclear Data Tables 20 397-439

[38] Fournier K B, May M J, Pacella D, Finkenthal M, Gregory B C and Goldstein W H 2000 Nuclear Fusion 40 847-864

[39] Mattioli M, Mazzitelli G, Fournier K B, Finkenthal M and Carraro L 2006 Journal of Physics B: Atomic, Molecular and Optical Physics 39 4457-4489

[40] Hollmann E M, Parks P B and Scott H A 2008 Contrib. Plasma Phys. 48 260-264

[41] Yoshino R, Tokuda S and Kawano Y 1999 Nucl. Fusion 39 151-161

[42] Yoshino R and Tokuda S 2000 Nucl. Fusion 40 1293-1309

[43] Tamai H, Yoshino R, Tokuda S, Kurita G, Neyatani Y, Bakhtiari M, Khayrutdinov R R, Lukash 
V E, Rosenbluth M M and JT-60 Team 2002 Nucl. Fusion 42 290-294 